\title{
Severe Imported Falciparum Malaria: A Cohort Study in 400 Critically III Adults
}

\author{
Fabrice Bruneel ${ }^{1 *}$, Florence Tubach ${ }^{2,3}$, Philippe Corne ${ }^{4}$, Bruno Megarbane ${ }^{5}$, Jean-Paul Mira ${ }^{6}$, Eric Peytel ${ }^{7}$, \\ Christophe Camus ${ }^{8}$, Frederique Schortgen ${ }^{9}$, Elie Azoulay ${ }^{10}$, Yves Cohen ${ }^{11}$, Hugues Georges ${ }^{12}$, Agnes \\ Meybeck $^{13}$, Herve Hyvernat ${ }^{14}$, Jean-Louis Trouillet $^{15}$, Eric Frenoy ${ }^{16}$, Laurent Nicolet ${ }^{17}$, Carine Roy ${ }^{2,3}$, \\ Remy Durand ${ }^{18}$, Jacques Le Bras ${ }^{19}$, Michel Wolff ${ }^{20}$, Severe Imported Malaria in Adults (SIMA) Study \\ Group
}

1 Service de Reanimation, Centre Hospitalier de Versailles, Le Chesnay, France, 2 AP-HP, Hopital Bichat-Claude Bernard, Departement d'Epidemiologie, Biostatistique et Recherche Clinique, Paris, France, 3 Institut National de la Sante et de la Recherche Medicale, CIE 801, Paris, France, 4 Service de Reanimation, Centre Hospitalier Universitaire Gui de Chauillac, Montpellier, France, $\mathbf{5}$ Service de Reanimation Medicale et Toxicologique, Centre Hospitalier Universitaire Lariboisière, Assistance PubliqueHopitaux de Paris, Paris, France, 6 Service de Reanimation, Centre Hospitalier Universitaire Cochin Saint Vincent de Paul, Assistance Publique-Hopitaux de Paris, Paris, France, 7 Service de Reanimation, Hopital d'Instruction des Armées Laveran, Marseille, France, $\mathbf{8}$ Service de Reanimation, Centre Hospitalier Universitaire Pontchaillou, Rennes, France, 9 Service de Reanimation, Centre Hospitalier Universitaire Henri Mondor, Assistance Publique-Hopitaux de Paris, Créteil, France, 10 Service de Reanimation, Centre Hospitalier Universitaire Saint Louis, Assistance Publique-Hopitaux de Paris, Paris, France, 11 Service de Reanimation, Centre Hospitalier Universitaire Avicenne, Assistance Publique-Hopitaux de Paris, Bobigny, France, 12 Service de Reanimation, Centre Hospitalier de Tourcoing, Tourcoing, France, 13 Service de Reanimation, Centre Hospitalier Universitaire Louis Mourier, Assistance Publique-Hopitaux de Paris, Colombes, France, 14 Service de Reanimation, Centre Hospitalier Universitaire de Nice, Nice, France, 15 Service de Reanimation, Centre Hospitalier Universitaire Pitié-Salpêtriere, Assistance Publique-Hopitaux de Paris, Paris, France, 16 Service de Reanimation, Centre Hospitalier Universitaire d'Angers, Angers, France, 17 Service de Reanimation, Centre Hospitalier Universitaire de Nantes, Nantes, France, 18 Service de Parasitologie, Centre Hospitalier Universitaire Avicenne, Assistance Publique-Hopitaux de Paris, Bobigny, France, 19 Service de Parasitologie, Centre Hospitalier Universitaire Bichat-Claude Bernard, Assistance Publique-Hopitaux de Paris, Paris, France, $\mathbf{2 0}$ Service de Reanimation, Centre Hospitalier Universitaire BichatClaude Bernard, Assistance Publique-Hopitaux de Paris, Paris, France

\begin{abstract}
Background: Large studies on severe imported malaria in non-endemic industrialized countries are lacking. We sought to describe the clinical spectrum of severe imported malaria in French adults and to identify risk factors for mortality at admission to the intensive care unit.

Methodology and Principal Findings: Retrospective review of severe Plasmodium falciparum malaria episodes according to the 2000 World Health Organization definition and requiring admission to the intensive care unit. Data were collected from medical charts using standardised case-report forms, in 45 French intensive care units in 2000-2006. Risk factors for inhospital mortality were identified by univariate and multivariate analyses. Data from 400 adults admitted to the intensive care unit were analysed, representing the largest series of severe imported malaria to date. Median age was 45 years; $60 \%$ of patients were white, $96 \%$ acquired the disease in sub-Saharan Africa, and $65 \%$ had not taken antimalarial chemoprophylaxis. Curative quinine treatment was used in $97 \%$ of patients. Intensive care unit mortality was $10.5 \%$ (42 deaths). By multivariate analysis, three variables at intensive care unit admission were independently associated with hospital death: older age (per 10 -year increment, odds ratio [OR], 1.72; 95\% confidence interval $[95 \% \mathrm{Cl}], 1.28-2.32 ; P=0.0004)$, Glasgow Coma Scale score (per 1-point decrease, OR, 1.32; $95 \% \mathrm{Cl}, 1.20-1.45 ; P<0.0001$ ), and higher parasitemia (per $5 \%$ increment, $\mathrm{OR}, 1.41 ; 95 \% \mathrm{Cl}$, $1.22-1.62 ; P<0.0001)$.
\end{abstract}

Conclusions and Significance: In a large population of adults treated in a non-endemic industrialized country, severe malaria still carried a high mortality rate. Our data, including predictors of death, can probably be generalized to other nonendemic countries where high-quality healthcare is available.

Citation: Bruneel F, Tubach F, Corne P, Megarbane B, Mira J-P, et al. (2010) Severe Imported Falciparum Malaria: A Cohort Study in 400 Critically III Adults. PLoS ONE 5(10): e13236. doi:10.1371/journal.pone.0013236

Editor: Photini Sinnis, New York University School of Medicine, United States of America

Received May 19, 2010; Accepted September 13, 2010; Published October 8, 2010

Copyright: ๑ 2010 Bruneel et al. This is an open-access article distributed under the terms of the Creative Commons Attribution License, which permits unrestricted use, distribution, and reproduction in any medium, provided the original author and source are credited.

Funding: The authors have no support or funding to report.

Competing Interests: The authors have declared that no competing interests exist.

*E-mail: fbruneel@ch-versailles.fr

- The SIMA Study Group members are listed in the Acknowledgments section.

\section{Introduction}

Plasmodium falciparum malaria remains a major public health problem in endemic areas, with more than 1,000,000 deaths each year. About 12,000 cases of imported malaria are reported annually in Europe [1] and about 1300 in the United States [2]. Among non-endemic countries, France has the highest number of cases, about 5000 per year, of which 20 to 30 are fatal [3]; the 
second highest number is found in the United Kingdom, where the incidence is rising [4].

Severe imported malaria still carries a high mortality rate, which is estimated at $10 \%$ to $15 \%$, although large studies are lacking [3]. One of the objectives of the World Health Organization (WHO) program for malaria control in Europe is to identify risk factors for death that can be used to optimize treatment decisions. In 2003, we reported several risk factors identified in 188 patients admitted to a single French intensive care unit (ICU) in 1988-1999 [5]. Although this study was the largest on managing severe malaria in a non-endemic industrialized country, it was conducted in a single center, over a long period, and used the 1990 WHO definition of severe malaria [6]. The WHO issued a new definition in 2000 [7]. Moreover, new treatment strategies for severe sepsis have been introduced over the last decade.

The objectives of this study were to gather multicenter data about severe imported malaria managed in the ICU in recent years, and to identify risk factors for mortality present at ICU admission.

\section{Methods}

\section{Ethics Statement}

Our study was approved by the Ethics Committee of the French Society for Critical Care Medicine (approval \#07-211). Due to the retrospective design of the study, we did not obtain informed consent from the included patients. Nevertheless, all the data collected retrospectively were anonymized in a standardized casereport form and in the database.

\section{Study sites and population}

The study was performed in the 45 adult ICUs in France that constitute the SIMA Study Group of 30 civilian university hospitals, three military teaching hospitals, and 12 regional nonteaching hospitals. Consecutive adults admitted to the 45 ICUs with a diagnosis of severe falciparum malaria between January 2000 and October 2006 were included and data were collected retrospectively (in 2006-2007) from the medical charts using standardized case-report forms.

\section{Definitions}

According to the 2000 WHO definition [7], severe imported malaria was defined in this study as the combination of, (i) asexual $P$. falciparum forms in blood or a positive result of the $P$. falciparum histidine-rich protein 2 antigen-based rapid test, and (ii) one or more severity criteria (Table 1) at admission or within the first 2 days, and (iii) requirement of ICU admission (at the discretion of the ICU physicians). The $2000 \mathrm{WHO}$ definition of severe malaria [7] was modified in part according to the SEAQUAMAT group [8]. More specifically, respiratory distress criteria were defined as stated in Table 1. Moreover, and to better characterize lung injury, we also defined respiratory failure as follows: in spontaneously breathing patients, $\mathrm{PaO}_{2}<60 \mathrm{mmHg}$ on room air or need for supplemental oxygen and/or respiratory rate $>32 /$ min; and in patients receiving ventilatory assistance, $\mathrm{PaO}_{2} / \mathrm{FiO}_{2}$ ratio $<200 \mathrm{mmHg}$.

Nonimmune patients were defined as Caucasians who traveled occasionally to endemic areas [5].

Community-acquired co-infection was defined as any infection diagnosed within the first 2 days after hospital admission. Infections occurring later were considered nosocomial. Acute lung injury (ALI) and acute respiratory distress syndrome (ARDS) were defined according to Bernard et al. [9].

\section{Management}

Treatment, particularly the management of organ dysfunctions (including levels for blood products transfusions), was at the discretion of the ICU physicians. During the study period, recent and detailed French guidelines for the management of severe

Table 1. Clinical and biological criteria for severe malaria according to the 2000 World Health Organization definition with modifications (see ${ }^{*}$ and $\dagger$ ).

\section{Clinical criteria}

Impaired consciousness: Glasgow Coma Scale score $<11^{*}$

Respiratory distress: requirement for noninvasive and/or endotracheal mechanical ventilation or spontaneous breathing with $\mathrm{PaO} \mathrm{O}_{2}<60 \mathrm{~mm} \mathrm{Hg}$ (if $\mathrm{FiO}_{2} \geq 0.21$ ) $\dagger$, and/or respiratory rate $>32 / \mathrm{min}^{*}$

Multiple convulsions

Circulatory collapse: systolic blood pressure $<80 \mathrm{~mm} \mathrm{Hg}$ despite adequate volume repletion

Abnormal bleeding

Jaundice: clinical jaundice or bilirubin $>50 \mu \mathrm{mol} / \mathrm{L}$

Macroscopic hemoglobinuria: if unequivocally related to acute malaria (patients with blackwater fever were not included)

Laboratory criteria

Severe anemia: hemoglobin $<5 \mathrm{~g} / \mathrm{dL}$

Hypoglycemia: blood glucose $<2.2 \mathrm{mmol} / \mathrm{L}$

Acidemia $(\mathrm{pH}<7.35)$ or acidosis (serum bicarbonate $<15 \mathrm{mmol} / \mathrm{L}$ )

Hyperlactatemia: arterial lactate $>5 \mathrm{mmol} / \mathrm{L}$

Hyperparasitemia $\geq 4 \%$

Renal impairment: serum creatinine $>265 \mu \mathrm{mol} / \mathrm{L}$ or blood urea nitrogen $>17 \mathrm{mmol} / \mathrm{L}^{*}$

* Coma scale criteria of 11 instead of 9; respiratory rate $>32 /$ minute and blood urea nitrogen $>17$ mmol/L are modifications according to the SEAQUAMAT group [8]. ${ }^{\dagger}$ The requirement for noninvasive and/or endotracheal mechanical ventilation or spontaneous breathing with $\mathrm{PaO}_{2}<60 \mathrm{~mm} \mathrm{Hg}$ (if $\mathrm{FiO}_{2} \geq 0.21$ ) was used specifically for this study.

doi:10.1371/journal.pone.0013236.t001 
falciparum malaria were available [10]. These guidelines strongly recommended a quinine loading dose but did not recommend exchange transfusion.

Blood culture for microbiology and chest X-ray were performed routinely at ICU admission. Neuroimaging, electroencephalogram and hemodynamic investigations were performed if deemed necessary by the attending physician.

\section{Data collection}

We recorded demographic data; previous medical history; country of malaria acquisition; chemoprophylaxis; clinical, laboratory, and imaging findings; and treatments and vital status at ICU and hospital discharge. Severity at ICU admission was assessed by computing the Glasgow Coma Scale (GCS) score [11], Simplified Acute Physiology Score II (SAPS II) [12], and Sequential Organ Failure Assessment (SOFA) score [13]. In each ICU, one intensivist completed a standardized case-report form for each patient. All case-report forms were reviewed by one of us (FB) for identification and resolution of inconsistencies.

\section{Statistical analysis}

A descriptive analysis was performed on the overall sample. Categorical variables were described with numbers and percentages and continuous variables with mean and $\mathrm{SD}$, or with median and interquartile range [IQR] for nonnormally distributed variables. Survivors and nonsurvivors were compared using the chi-square test for categorical variables and the Student's t-test or Wilcoxon rank-sum test, as appropriate, for continuous variables.

To identify risk factors for mortality, we constructed logistic regression models using a combination of multiple imputations and bootstrapping to select prognostic variables and to handle missing data [14]. Potential risk factors were as follows (*worst value during the 24 hours after ICU admission): sex, ethnic origin, co-morbidities, pulmonary failure*, shock*, age, time from symptom onset to ICU admission, GCS score*, plasma bicarbonate*, hemoglobin*, leukocytes*, platelets*, prothrombin time*, plasma creatinine*, highest blood glucose*, lowest blood glucose*, total serum bilirubin*, ALAT*, and parasitemia*. From the initial data set, we constructed 1000 bootstrap data sets and performed automatic stepwise logistic regression in each, selecting variables associated with mortality with $P<20 \%$ by univariate analysis. Then, variables were selected for the second step if they were associated with mortality with $P$ values $<20 \%$ in at least $20 \%$ of the 1000 models. We implemented multiple imputation of missing data, which yielded five data sets. We then constructed 1000 bootstrap data sets for each imputed data set (i.e., 5000 data sets) and performed automatic stepwise logistic regression in each. Variables present in at least $60 \%$ of the 5000 models were selected for the final model. In the last step, we used the mean of the coefficient estimations obtained in each of the five imputed data sets. SAS 9.1 software (SAS Inc, Cary, NG) was used for all statistical analyses.

\section{Results}

During the study period, 400 patients admitted to the ICU for severe falciparum malaria, were included. The median number of patients per center was 7 (IQR, 5-10; range, 2 to 35).

\section{General characteristics of the 400 patients}

These characteristics are reported according to survival status in Table 2. Falciparum malaria was acquired in sub-Saharan Africa in $95.5 \%$ of the 366 patients for whom this information was available. Median stay duration in the endemic area was 1.0 month (IQR, 0.5-2.0). Of the $34.7 \%$ of patients who reported taking anti-malarial chemoprophylaxis, only $45.5 \%$ reported good adherence. Conditions associated with immune deficiency were noted in $7.3 \%$ of patients. One or more co-morbidities were present in $14.3 \%$ of patients. Five patients were pregnant.

\section{Baseline patient characteristics and antimalarial therapy at ICU admission}

The main clinical and laboratory characteristics and the pattern of WHO severity criteria during the first 24 hours in the ICU in survivors and nonsurvivors are reported in Tables 3 and 4, respectively.

Overall, median parasitemia at ICU admission was $7.0 \%$ IQR, $2.7-15.0 \%)$. P. falciparum was identified in thin blood films in $84.7 \%$ of patients and/or in thick blood films in $50.7 \%$ and/or by antigen detection in $11.5 \%$.

Intravenous quinine was used in $391(97.8 \%)$ patients and other antimalarials in 9 patients. A quinine loading dose was used in 244 $(61 \%)$ patients. The mean loading dose was $1072 \pm 359 \mathrm{mg}$, consistent with the mean body weight $(72.7 \pm 14.5 \mathrm{~kg})$ and French

Table 2. General characteristics of the 400 patients admitted to the intensive care unit with severe malaria by survival status.

\begin{tabular}{|c|c|c|c|}
\hline Parameter & Survivors $n=358$ & Nonsurvivors $n=42$ & $P$ value* \\
\hline Mean age $( \pm S D)$, years & $42.8 \pm 15.0$ & $55.6 \pm 14.2$ & $<0.0001$ \\
\hline Male, \% & 68.3 & 83.3 & 0.04 \\
\hline White, \% & 57.7 & 79.5 & 0.01 \\
\hline Black, \% & 36.0 & 12.8 & \\
\hline Other, \% & 6.3 & 7.7 & \\
\hline Previous malaria episodes, $\%$ & 23.4 & 17.1 & 0.36 \\
\hline Antimalarial chemoprophylaxis, \% & 34.8 & 34.1 & 0.94 \\
\hline Nonimmune patients, \% & 43.8 & 59.5 & 0.05 \\
\hline At least one co-morbidity, $\%$ & 13.1 & 23.8 & 0.06 \\
\hline Immune deficiency, \% & 7.8 & 2.6 & 0.34 \\
\hline Mean time from symptom onset to ICU admission ( \pm SD), days & $5.4 \pm 5.1$ & $6.2 \pm 4.6$ & 0.14 \\
\hline
\end{tabular}


Table 3. Baseline characteristics at intensive care unit admission in the 400 patients by survival status.

\begin{tabular}{|c|c|c|c|}
\hline Parameter & Survivors $n=358$ & Nonsurvivors $n=42$ & $P$ value* \\
\hline SAPS II, mean \pm SD & $30.2 \pm 15.6$ & $70.6 \pm 26.3$ & $<0.0001$ \\
\hline SOFA score, mean $\pm S D$ & $7.7 \pm 3.8$ & $15.4 \pm 5.2$ & $<0.0001$ \\
\hline Glasgow Coma Scale score, median [IQR] & $15.0[12.0-15.0]$ & $9.0[3.0-14.0]$ & $<0.0001$ \\
\hline Respiratory failure \% & $13.4 \%$ & $45.2 \%$ & $<0.0001$ \\
\hline Highest temperature in ${ }^{\circ} \mathrm{C}$, mean $\pm \mathrm{SD}$ & $39.0 \pm 1.1$ & $38.7 \pm 1.1$ & 0.08 \\
\hline Arterial $\mathrm{pH}$, mean $\pm \mathrm{SD}$ & $7.41 \pm 0.1$ & $7.19 \pm 0.2$ & $<0.0001$ \\
\hline Serum bicarbonates in $\mathrm{mmol} / \mathrm{L}$, mean $\pm \mathrm{SD}$ & $21.7 \pm 4.7$ & $15.7 \pm 7.2$ & $<0.0001$ \\
\hline Arterial lactate in mmol/L, median [IQR] & $2.4[1.6-4.0]$ & $8.4[4.5-16.0]$ & $<0.0001$ \\
\hline Hemoglobin in $\mathrm{g} / \mathrm{dL}$, mean $\pm S D$ & $10.3 \pm 2.6$ & $9.2 \pm 2.9$ & 0.01 \\
\hline $\mathrm{WBC} \cdot 10^{3} / \mathrm{mm}^{3}$, median $[\mathrm{IQR}]$ & $6.4[4.7-9.6]$ & $9.7[5.9-17.9]$ & 0.004 \\
\hline Platelet count $\cdot 10^{3} / \mathrm{mm}^{3}$, median [IQR] & $35.0[19.0-57.0]$ & $19.0[13.0-29.0]$ & $<0.0001$ \\
\hline Plasma prothrombin time in $\%$, mean $\pm S D$ & $72.6 \pm 16.8$ & $53.4 \pm 26.8$ & $<0.0001$ \\
\hline Serum creatinine in $\mu \mathrm{mol} / \mathrm{L}$, median $[\mathrm{IQR}]$ & $117.0[87.0-228.0]$ & $203.0[156.0-302.0]$ & $<0.0001$ \\
\hline Lowest serum glucose in $\mathrm{mmol} / \mathrm{L}$, mean $\pm \mathrm{SD}$ & $5.4 \pm 2.1$ & $4.7 \pm 2.4$ & 0.03 \\
\hline Highest serum glucose in $\mathrm{mmol} / \mathrm{L}$, mean $\pm \mathrm{SD}$ & $9.6 \pm 4.5$ & $12.3 \pm 5.9$ & 0.0002 \\
\hline Total bilirubin in $\mu \mathrm{mol} / \mathrm{L}$, median $[\mathrm{IQR}]$ & $51.5[28.0-82.5]$ & $99.0[47.0-181.0]$ & $<0.0001$ \\
\hline Parasitemia first day in \%, median [IQR] & $6.0[2.0-14.0]$ & $17.0[7.5-30.0]$ & $<0.0001$ \\
\hline Serum sodium in $\mathrm{mmol} / \mathrm{L}$, mean $\pm \mathrm{SD}$ & $131.9 \pm 5.8$ & $132.4 \pm 9.2$ & 0.90 \\
\hline Serum potassium in $\mathrm{mmol} / \mathrm{L}$, mean $\pm S D$ & $3.8 \pm 0.6$ & $4.1 \pm 1.1$ & 0.09 \\
\hline Serum ALAT in IU, median [IQR] & $58.0[35.0-108.0]$ & $104.5[68.0-261.0]$ & $<0.0001$ \\
\hline Serum lactate dehydrogenase in IU, median [IQR] & $966.0[600.0-1428.0]$ & 2239.5 [1121.0-4128.0] & $<0.0001$ \\
\hline Serum C-reactive protein in $\mathrm{mg} / \mathrm{L}$, mean $\pm \mathrm{SD}$ & $165.2 \pm 86.8$ & $198.8 \pm 93.7$ & 0.06 \\
\hline
\end{tabular}

Table 4. Severity criteria in the 400 patients at intensive care unit admission, according to outcome.

\begin{tabular}{|c|c|c|c|}
\hline Severity criteria & Survivors $n=358$ & Nonsurvivors $n=42$ & $P$ value* \\
\hline Impaired consciousness, n (\%) & $77(21.5)$ & $26(62.0)$ & $<0.0001$ \\
\hline Respiratory distress, n (\%) & $72(20.1)$ & $29(69.1)$ & $<0.0001$ \\
\hline Multiple convulsions, n (\%) & $22(6.2)$ & $5(11.9)$ & 0.1849 \\
\hline Shock, n (\%) & $69(19.3)$ & $26(61.9)$ & $<0.0001$ \\
\hline Abnormal bleeding, n (\%) & $7(2.0)$ & $3(7.1)$ & 0.0766 \\
\hline Jaundice, n (\%) & $178(49.7)$ & $30(71.4)$ & 0.0077 \\
\hline Hemoglobinuria, n (\%) & $20(5.6)$ & $2(4.8)$ & 1.0000 \\
\hline Severe anemia, n (\%) & $10(2.8)$ & $4(9.5)$ & 0.0453 \\
\hline Hypoglycemia, n (\%) & $5(1.4)$ & $8(19.1)$ & $<0.0001$ \\
\hline Acidosis, n (\%) & $43(12.0)$ & $27(64.3)$ & $<0.0001$ \\
\hline Hyperlactatemia, n (\%) & $37(10.3)$ & $26(61.9)$ & $<0.0001$ \\
\hline Parasitemia > 4\%, n (\%) & $223(62.3)$ & $34(81.0)$ & 0.0170 \\
\hline Renal impairment, n (\%) & $111(31.0)$ & $26(61.9)$ & $<0.0001$ \\
\hline Mean number of severity criteria during the first 24 hours in the ICU ( $\pm S D$ ) & $2.4 \pm 1.6$ & $5.9 \pm 2.4$ & $<0.0001$ \\
\hline
\end{tabular}

WHO, World Health Organization; ICU, intensive care unit.

*according to univariate analysis.

doi:10.1371/journal.pone.0013236.t004 
guidelines (quinine loading dose, $16 \mathrm{mg} / \mathrm{kg}$ ) [9]. Of the 156 patients who received no loading dose, 9 did not receive quinine, 90 had received curative antimalarial treatment within hours before ICU admission, and 57 were not considered to require a loading dose (less severe form of malaria according to the physician in charge). An antibiotic was added in 36 patients. No patient underwent exchange transfusion.

\section{Complications of malaria and management}

In 97 (24.3\%) patients, at least one WHO defining criterion that was absent during the first 24 hours developed over the next 48 hours: impaired consciousness $(n=20)$, respiratory distress $(\mathrm{n}=45)$, multiple convulsions $(\mathrm{n}=6)$, shock $(\mathrm{n}=26)$, abnormal bleeding $(n=6)$, jaundice $(n=12)$, hemoglobinuria $(n=2)$, severe anemia $(n=6)$, hypoglycemia $(n=5)$, acidosis $(n=10)$, hyperlactatemia $(n=7)$, hyperparasitemia $(n=4)$, and renal function impairment $(\mathrm{n}=23)$.

Consciousness was impaired in 123 patients (103 at admission), for a mean duration of 2 days (IQR, 1-6; range, 1-67). Convulsions occurred in 33 patients, of whom 21 needed anticonvulsant therapy. Focal neurological abnormalities were present in 22 patients. Cerebral computed tomography (CT) and/ or magnetic resonance imaging (MRI) were performed in 76 and 13 patients, respectively, and the findings were abnormal in 23 patients. At ICU discharge, 24 patients had at least one residual neurological abnormality: mental status abnormalities $(n=12)$, persistent focal deficits $(\mathrm{n}=8)$, critical-illness polyneuropathy $(n=6)$, epilepsy $(n=2)$, and other abnormalities $(n=7)$.

On the first day, fluid resuscitation was given to $247(61.7 \%)$ patients, including $213(213 / 358,59.5 \%)$ survivors and 34 (34/42, $81.0 \%)$ nonsurvivors $(P=0.0068)$. Throughout the ICU stay, 109 patients received catecholamines (norepinephrine, $\mathrm{n}=81$; and/or epinephrine, $\mathrm{n}=26$; and/or dopamine; $\mathrm{n}=27$; and/or dobutamine, $\mathrm{n}=25)$, including $71(71 / 358,19.8 \%)$ survivors and 38 (38/ $42,90.5 \%)$ nonsurvivors $(P<0.0001)$.Low-dose steroids were used in 43 patients and activated protein $\mathrm{C}$ in only 3 patients.

At least one co-infection was diagnosed during the ICU stay in 96 patients $(24.0 \%)$, including $78(78 / 358,21.8 \%)$ survivors and $18(18 / 42,42.9 \%)$ nonsurvivors $(P=0.0025)$. Community-acquired bacterial infections were diagnosed in $30(30 / 96,31.2 \%)$ patients including $25(25 / 358,7.0 \%)$ survivors and $5(5 / 42$, $11.9 \%)$ nonsurvivors $(P=0.2520)$. In the remaining $66(66 / 96$,
$68.8 \%$ ) patients with bacterial infections, the first episode was nosocomially acquired; 53 (53/358, 14.8\%) of these patients were among the survivors and $13(13 / 42,31.0 \%)$ among the nonsurvivors $(P=0.0076)$. The main co-infection sites and microorganisms are detailed in the Table 5 .

Endotracheal mechanical ventilation was required in 116 (116/ $395,29.4 \%$ ) patients, for a median duration of 6 days (IQR, 3-14; range, 1-201). In addition, 32 patients received noninvasive ventilation, which failed in 16 patients, who then received endotracheal mechanical ventilation. Thus, mechanical ventilation was used in 132 patients, of whom 90 survived and 42 died. Nonhemodynamic pulmonary edema was present in $76(57.5 \%)$ of the 132 ventilated patients; among them, 58 had ARDS and 18 had ALI. The 56 other patients received ventilatory assistance essentially because of unarousable coma.

During the ICU stay, 81 patients required renal replacement therapy (intermittent hemodialysis, $\mathrm{n}=64$; and/or continuous veno-venous hemodiafiltration, $\mathrm{n}=49$ ).

Abnormal bleeding occurred in 23 patients. Blood transfusions were required in 114 patients, platelets in 63, and fresh plasma in 24. Overall, 845 blood-product units were transfused (538 blood, 177 platelets, and 130 fresh plasma units).

\section{Outcomes and factors predicting death}

The overall hospital mortality rate was $10.5 \%$ (95\% confidence interval [95\%CI], 7.5\%-13.5\%), because all 42 deaths occurred in the ICU. The main characteristics of the nonsurvivors and causes of death are shown in Table S1. Death occurred within the first ICU week in $32(76.2 \%)$ patients. Median ICU stay duration in the 42 patients who died was 3.5 days (IQR, 2-7; range, 1-198). Median ICU and hospital stay durations in survivors were 5 days (IQR, 3-8; range, 1-186) and 10 days (IQR, 7-17; range, 1-189), respectively.

By multivariable analysis, three variables present within 24 hours after ICU admission were significantly associated with death in the ICU: older age, lower Glasgow Coma Scale score, and higher parasitemia (Table 6).

\section{Discussion}

In 400 adults with severe imported malaria, ICU mortality was $10.5 \%$. Three variables present at ICU admission independently predicted death: older age, coma, and higher parasitemia.

Table 5. Data on the 96 first episodes of co-infection in the 400 adults with severe imported malaria.

\begin{tabular}{|c|c|c|}
\hline Parameter & Community-acquired infections $n=30$ & Nosocomial infections $n=66$ \\
\hline $\begin{array}{l}\text { Pneumonia } \\
\text { Microorganisms } \\
\text { (number of episodes) }\end{array}$ & $\begin{array}{l}13 \text { episodes } \\
\text { Streptococcus pneumoniae (3), MS Staphylococcus aureus } \\
\text { (3), Gram+ cocci (negative culture) (1), Escherichia coli (1), } \\
\text { Haemophilus influenzae (1), Klebsiella pneumoniae (1), } \\
\text { Enterobacter cloacae (1), Acinetobacter baumannii (1), } \\
\text { Pseudomonas aeruginosa (1), Not documented (2) }\end{array}$ & $\begin{array}{l}48 \text { episodes } \\
\text { MS S. aureus (11), Streptococcus sp (7), S. pneumoniae (3), MR S. } \\
\text { aureus (1), H. influenzae (9), E. coli (4), K. pneumoniae (1), } \\
\text { Enterobacter aerogenes (3), E. cloacae (2), A. baumannii (3), P. } \\
\text { aeruginosa (5), Bulkholderia cepacia (1), Legionella pneumophila } \\
\text { (1), Citrobacter koseri (1), Not documented (6) }\end{array}$ \\
\hline $\begin{array}{l}\text { Bacteremia } \\
\text { Microorganisms } \\
\text { (number of episodes) }\end{array}$ & $\begin{array}{l}10 \text { episodes } \\
\text { E. coli (3), P. aeruginosa (1), Alcaligenes xylosoxidans (1), } \\
\text { Clostridium sp (1), K. pneumoniae (1), Salmonella typhi (1), } \\
\text { Campylobacter jejuni (1), Candida albicans (1) }\end{array}$ & $\begin{array}{l}8 \text { episodes } \\
\text { E. coli (4), MS S. aureus (1), Staphylococcus epidermidis (1), } \\
\text { Klebsiella oxytoca (1), S. typhi (1) }\end{array}$ \\
\hline $\begin{array}{l}\text { Urinary tract infection } \\
\text { Microorganisms } \\
\text { (number of episodes) }\end{array}$ & $\begin{array}{l}3 \text { episodes } \\
\text { E. coli (2), Proteus sp (1) }\end{array}$ & $\begin{array}{l}9 \text { episodes } \\
\text { E. coli (5), K. pneumoniae (3), Enterococcus sp ( } 2 \text { ) }\end{array}$ \\
\hline $\begin{array}{l}\text { Other sites of infection } \\
\text { (number of episodes) }\end{array}$ & Abdominal infection (3), Skin and soft tissue (1) & Catheter infection (3), Sinusitis (2) \\
\hline
\end{tabular}


Table 6. Independent predictors of death at intensive care unit admission in the 400 patients.

\begin{tabular}{llll}
\hline & N & OR (95\%Cl) & P value \\
\hline Age (per 10-year increment) & 400 & $1.72[1.28-2.32]$ & 0.0004 \\
$\begin{array}{l}\text { Glasgow Coma Scale score } \\
\text { (per 1-point increment) }\end{array}$ & 400 & $1.32[1.20-1.45]$ & $<0.0001$ \\
$\begin{array}{l}\text { Parasitemia (per 5\% increment) } \\
\text { R of the model: 0.90. }\end{array}$ & 400 & $1.41[1.22-1.62]$ & $<0.0001$ \\
\hline $\begin{array}{l}\text { OR, odds ratio; 95\%Cl, 95\% confidence interval. } \\
\text { doi:10.1371/journal.pone.0013236.t006 }\end{array}$ & & \\
\end{tabular}

To the best of our knowledge, this is the largest study of severe imported falciparum malaria in adults managed in a nonendemic country with high-level intensive-care facilities. It provides useful insights on disease outcomes and variables contributing to mortality, at least in France. These results should prove useful to clinicians managing severe malaria patients in the ICU, as well as to epidemiologists and public health practitioners studying potential risk factors for mortality in severe imported malaria. They may help to provide recommendations for intensivists, especially in countries where imported malaria is uncommon.

Whether our results can be generalized to other nonendemic countries deserves discussion. The few recent studies on severe imported malaria cannot be compared directly, for multiple reasons. Nevertheless, a number of points are shared by our study and recent studies from the UK and US $[2,4,15,16]$ : the majority of severe cases are related to $P$. falciparum, stay duration in endemic areas is around 20-30 days [4,16], and fewer than $15 \%$ of patients take adequate chemoprophylaxis $[2,4,15]$. These common points suggest that our results may be at least partly relevant to other nonendemic countries having similar levels of intensive care. Finally, our results may serve as a reference for achievable survival rates and evaluations of complication rates in nonimmune individuals in resource-poor settings, where the investigations needed to characterize complications may be unavailable (e.g., routine high-quality chest X-ray, neuro-imaging studies, routine blood cultures, and hemodynamic measurements).

The definition of severe falciparum malaria issued by the WHO in 2000 is accompanied with data on the frequency and prognostic value of the defining criteria [7]. However, few such data are available for adults with severe imported malaria [3]. In our cohort, neither seizure activity nor hemoglobinuria was significantly associated with death in the univariate analysis. Among the other criteria, bleeding, anemia, and hypoglycemia are very uncommon in adults, and their prognostic relevance is therefore unclear. In our univariate analysis, six WHO criteria present within 24 hours of ICU admission were common and strongly correlated with ICU mortality, namely, impaired consciousness, respiratory distress, shock, metabolic acidosis, hyperlactatemia, and renal failure. Parasitemia $>4 \%$ and jaundice were the two most common WHO criteria but were less strongly associated with mortality. We compared the main WHO severity criteria at admission in our 400 adults (including 182 nonimmune patients) and in 1050 Asian patients (including 11\% young children and $26 \%$ older children) from low-transmission areas [17]. Globally, our population had (1) lower rates of coma, convulsions, acidosis, respiratory distress, and anemia; (2) similar rates of jaundice and renal failure; and (3) higher rates of shock and hyperparasitemia. Nevertheless, a large number of factors can influence the presentation, particularly access to healthcare (and more specifically to high-level intensive care) and time from symptom onset to hospital admission. Furthermore, the characteristics of the compared populations are often noticeably different in terms of age, proportion of pediatric patients, ethnic origin, level of antimalaria immunity, background health status, and living standards.

Severe imported malaria continues to carry a high mortality rate even when high-quality healthcare is available and the affected population is chiefly composed of healthy adults. In our previous single-center retrospective study, mortality was $11 \%$ in the 93 patients with severe disease [5]. Among 76 episodes of severe adult imported malaria seen in the UK in 1991-2006, 8\% were fatal [15]. These findings are consistent with our $10.5 \%$ mortality rate. In the two main trials of artemisinin derivatives versus quinine in adults with severe falciparum malaria in endemic areas, higher mortality rates of $15 \%$ versus $22 \%$, and $13 \%$ versus $17 \%$, respectively, were found $[8,18]$. Although populations in endemic and nonendemic areas are not comparable, we believe the lower mortality in our study than in the quinine groups of these two trials is mainly ascribable to the availability of optimal treatments for organ failures and to the better baseline health status of our population.

Our multivariable analysis identified three factors present at ICU admission and predictive of death. Older age independently predicted death, in keeping with recent data from both endemic and nonendemic areas $[17,19]$. This adverse impact of older age should receive careful attention given the increasing numbers of older travelers. Consciousness impairment assessed using the GCS score on the first ICU day also independently predicted death in our study. In our previous study, several factors, including impaired consciousness, were associated with death by univariate analysis, but the number of nonsurvivors was too small for a multivariable analysis [5]. Our findings in patients with severe imported malaria agree with the largest recent study conducted in endemic areas, which found that the main independent predictors of death were cerebral malaria (as assessed by the Glasgow Coma Score) and acidosis [20]. In contrast to studies from Africa and Asia, our study found no independent effect of acidosis at ICU admission on the risk of death. We included serum bicarbonate instead of arterial lactate in the multivariate analysis, because we had missing data on lactate levels, which probably contributed to the lack of significance of arterial lactate in the multivariate model. The missing lactate data can be ascribed chiefly to the retrospective study design, as arterial lactate was not measured routinely in patients at the lower end of the severity range, especially at the beginning of the study period. Unfortunately, base excess was not available on our case-report form. Nevertheless, bicarbonate and base excess, as measures of acidosis, were of roughly similar prognostic value, as shown in a recent study [20]. The third variable independently associated with death was parasitemia at ICU admission. The prognostic impact of parasitemia in severe malaria patients varies with multiple factors including geographic area, ethnicity, level of anti-malaria immunity, and previous exposure to antimalarial agents. Furthermore, parasitemia may chiefly reflect the circulating parasite load, which may not mirror the amount of parasites sequestered in the microvasculature. One group showed that total-body parasite biomass estimated from the plasma concentration of $P$. falciparum histidine-rich protein 2 (HRP2) predicted death more reliably than did parasitemia [21]. Few data are available on the prognostic significance of parasitemia in patients with severe imported malaria [3]. In our earlier study, median parasitemia was $18.2 \%$ in the 10 nonsurvivors and $3.5 \%$ in the 83 survivors $(P=0.02)$ but the difference was not significant after adjustment [5]. According to WHO guidelines, parasitemia $>4 \%$ is sufficient to indicate treatment for severe malaria [22]. In a recent study including 482 
cases of falciparum malaria, of which 72 were severe and 6 were fatal, the odds of having severe falciparum malaria were 12 times higher in patients with parasitemia $\geq 2 \%$ than in those with parasitemia $<2 \%$ [15]. Further studies are needed to clarify the relevance of both parasitemia and plasma P. falciparum HRP2 as predictors of death in adults with severe imported malaria.

Although an earlier study showed that ethnic origin was an independent risk factor for severe malaria [15], ethnic origin did not independently predict death in our study. We cannot rule out a weak association of ethnic origin with mortality in patients who already have severe malaria, and our sample size may have been too small to detect a statistically significant effect.

Finally, to better assess the relevance of co-infections, we included "at least one infection" in the multivariable model. This parameter was not significant, suggesting that it did not independently predict death. In our setting, co-infections seem associated with severity rather than independently responsible for increased severity.

Our study has strong points compared to the main recent studies of severe imported malaria $[5,15]$. The patient population is considerably larger and comes from 45 different centers, which increases the general validity of our findings. Furthermore, our study covers a recent period and therefore reflects the impact of the many recent advances in the management of severe sepsis $[23,24]$. Finally, nearly all the patients received quinine therapy and, therefore, our results were not confounded by differences in the antimalarial drugs used. One limitation of our study is the retrospective design. However, the database was established by collecting information on consecutive patients using standardized data-collection forms. To obtain an adequate recruitment rate, we had to select adult ICUs experienced in the management of severe malaria, and consequently only 45 ICUs participated in the study. Some data were missing from the database. Nevertheless, this point was carefully taken into account and corrected by the statistical methodology using both multiple imputation and bootstrapping [14].

Our findings suggest several targets for improving the management of severe imported malaria. First, inappropriate chemoprophylaxis was common among both survivors and nonsurvivors. Efforts to improve adherence to chemoprophylaxis regimens are clearly needed. Second, we identified several independent predictors of death. A more standardized and aggressive treatment approach to patients with these predictors (and in particular immediately upon the onset of neurological impairment) might decrease the mortality rate. Third, we used quinine in nearly all our patients. A large study of adults with severe malaria conducted recently in Asia found that artesunate was associated with lower mortality rates and better tolerance, compared to quinine [8]. Thus, it can be hoped that the introduction of intravenous artesunate in nonendemic areas will improve survival rates in patients with severe imported malaria [25]. Unfortunately, intravenous artesunate is not yet available in France or other European countries. The only data on artesunate therapy for severe imported falciparum malaria come from a letter about 9 Norwegian patients, all of whom achieved a full recovery [26], and from the 2008 report of the Centers for Disease Control on 24 patients, of whom 1 died [2]. According to all these data, we wish to have parenteral artesunate, produced according to good manufacturing practices, in Europe.

In conclusion, severe imported falciparum malaria in adults still carried a high mortality rate in patients who received quinine and optimal supportive care in the ICU. The strongest predictors of death at ICU admission were older age, neurological impairment, and high parasitemia. Patients with these factors may require the highest level of treatment intensity. The results of our multicenter study are probably relevant to other nonendemic countries where high-quality healthcare is available.

\section{Supporting Information}

Table S1 Nonsurvivors: Main characteristics and causes of death. Large table to describe the main characteristics and causes of death in the 42 nonsurvivors.

Found at: doi:10.1371/journal.pone.0013236.s001 (0.10 MB DOG)

\section{Acknowledgments}

We thank A. Wolfe MD for helping to prepare the manuscript.

We thank the nurses, physicians, and biologists of the SIMA Study Group (see infra) for their excellent care of the patients.

Collaborators of the SIMA Study Group (including authors)

Hopital Bichat Claude Bernard, Paris: M. Wolff and B. Regnier (ICU),

S. Houze and J. Le Bras (Parasitology)

Hopital Sant Andre, Bordeaux: O. Guisset and C. Gabinski (ICU), MC.

Receveur and P. Vincendeau (Parasitology)

Hopital d'Angers: E. Fresnoy and A. Mercat (ICU), L. de Gentile and D.

Chabasse (Parasitology)

Hopital de Belfort: O. Ruyer and JP. Faller (ICU), M. Laplace (Parasitology)

Hopital Paul Morel, Vesoul: P. Daoudal and C. Floriot (ICU), A. Royer (Parasitology) Hopital d'Orleans: I. Runge and T. Boulain (ICU), JM. Segalin and L. Bret (Parasitology) Hopital Henri Mondor, Creteil: M.

Fortin, F. Schortgen and C. Brun Buisson (ICU), F. Botterel and S. Bretagne (Parasitology)

Hopital de Meaux: A. Combes (ICU), A. Vincenot (Parasitology)

Hopital de Clermont-Ferrand: F. Thiolliere and B. Souweine (ICU), D.

Pons and J. Sirot (Parasitology)

Hopital Robert Debre, Reims: J. Cousson and A. Leon (ICU), D.

Toubas and I. Villena (Parasitology)

Hopital Edouard Herriot, Lyon: I. Mohammedi and D. Robert (ICU),

F. de Montbrisson and S. Picot (Parasitology)

Hopital de Tourcoing: H. Georges and B. Guery (ICU), P.Patoz and C. Dhennain (Parasitology)

Hopital Charles Nicolle, Rouen: D. Chakarian and G. Bonmarchand

(ICU), P. Abboud and L. Favennec (Parasitology)

Hopital Laveran, Marseille: C. Gil, E. Peytel and JP. Carpentier (ICU),

F. Simon, E. Garnotel and JL. Moalic (Parasitology)

Hopital Jean Verdier, Bondy: L. Tual and G. Dhonneur (ICU), A. Collignon and I. Poilane (Parasitology)

Hopital de la Croix Rousse, Lyon: T. Fassier and C. Guerin (ICU), F. de Montbrisson and S. Picot (Parasitology)

Hopital de la Pitie Salpetriere, Paris: JL. Trouillet and C. Gibert (ICU), M. Thellier, D. Mazier and M. Danis (Parasitology)

Hopital de Limoges: H. Gastinne (ICU), D. Azjenberg and ML Darde (Parasitology)

Hopital Antoine Beclere, Clamart: F. Jacobs and F. Brivet (ICU), AM. Koka (Parasitology) Hopital de Grenoble: JF. Timsit (ICU), D. Maubon and H. Pelloux (Parasitology)

Hopital Cochin, Paris: A. Mathonnet, JP. Mira and JF. Dhainault (ICU),

H. Yera and J. Dupouy-Camet (Parasitology)

Hopital André Mignot, Le Chesnay: F. Bruneel and JP. Bedos (ICU), O.

Eloy and P. Therond (Parasitology)

Hopital de Rangueil, Toulouse: P. Cougot (ICU), F. Benoit-Vical and JF. Magnaval (Parasitology)

Hopital Louis Pasteur, Chartres: K. Ouchenir and P. Kalfon (ICU), Z. Benseddik and A. Secher (Parasitology)

Hopital Emile Muller, Mulhouse: P. Guiot and Y. Mootien-Joy (ICU), A. Gravet and JM. Delarbre (Parasitology)

Hopital Ambroise Pare, Boulogne: B. Page and F. Jardin (ICU), J.

Dunand (Parasitology) Hopital Jean Mimoz, Besancon: JC. Navellou and

G. Capellier (ICU), L. Millon and F. Grenouillet (Parasitology)

Hopital de Saint Germain en Laye: JL. Ricome (ICU), Y. Giudicelli (Parasitology) 
Hopital Yves Le Foll, Saint Brieuc: A. Courte and G. Guivarch (ICU), L. Guezennec (Parasitology)

Hopital Begin, Saint Mande: N. Libert and JM. Rousseau (ICU), G. Rapp, JE. Pilo and JD. Cavallo (Parasitology)

Hopital Purpan, Toulouse: O. Angles and M. Genestal (ICU), JF. Magnaval (Parasitology) Hopital Hotel Dieu, Nantes: L. Nicolet, G. Guitton and D. Villers (ICU), F. Gay-Andrieu and M.Marjolet (Parasitology)

Hopital Pontchaillou, Rennes: C. Camus and R. Thomas (ICU), S. Chevrier and C. Guigen (Parasitology)

Hopital Lariboisiere, Paris: B. Megarbane and F. Baud (ICU), G. Gourmel and F. Derouin (Parasitology)

Hopital Saint Louis, Paris: E. Azoulay and B. Schlemmer (ICU), G. Sarfati and F. Derouin (Parasitology)

Hopital Gui de Chauliac, Montpellier: P. Corne and P. Jonquet (ICU),

D. Basset, JF Schwed and JP. Dedet (Parasitology)

Hopital Lapeyronie, Montpellier: K. Klouche and P. Beraud (ICU), JP. Cristol and JP. Dedet (Parasitology)

Hopital Pellegrin, Bordeaux: B. Herpe and Y. Castaing (ICU), P. Fialon and P. Vincendeau (Parasitology)

\section{References}

1. World Health Organization (2006) From malaria control to elimination in the WHO European region 2006-2015. Regional office for Europe, Copenhagen, Denmark.

2. Mali S, Steele S, Slutsker L, Arguin PM; Centers for Disease Control and Prevention (2010) Malaria surveillance-United States, 2008. MMWR Surveill Summ 59: 1-15.

3. Société de Pathologie Infectieuse de Langue Française; Collège des Universitaires de Maladies Infectieuses et Tropicales; Société Française de Médecine des Armées; Société Française de Parasitologie; Société Française de Pédiatrie; Société de Médecine des Voyages; Société de Pathologie Exotique; Société de Réanimation de Langue Française (2008) Management and prevention of imported Plasmodium falciparum malaria (Revision 2007 of the 1999 Consensus Conference). Med Mal Infect 38: 68-117.

4. Smith AD, Bradley DJ, Smith V, Blaze M, Behrens RH, et al. (2008) Imported malaria and high risks groups: observational study using UK surveillance data 1987-2006. BMJ 337: a120. doi:10.1136/bmj.a120.

5. Bruneel F, Hocqueloux L, Alberti C, Wolff M, Chevret S, et al. (2003) The clinical spectrum of severe imported malaria in the intensive care unit. Am J Respir Crit Care Med 167: 684-689.

6. World Health Organization (1990) Severe and complicated malaria. Trans R Soc Trop Med Hyg 84(Suppl. 2): S1-65.

7. World Health Organization (2000) Severe falciparum malaria. Trans R Soc Trop Med Hyg 94(Suppl. 1): S1-90.

8. Dondorp A, Nosten F, Stepniewska K, Day N, White N; South East Asian Quinine Artesunate Malaria Trial (SEAQUAMAT) group (2005) Artesunate versus quinine for treatment of severe falciparum malaria: a randomised trial. Lancet 366: 717-725.

9. Bernard GR, Artigas A, Brigham KL, Carlet J, Falke K, et al. (1994) The Consensus Committee. Report of the American-European conference on ARDS: definitions, mechanisms, relevant outcomes and clinical trial coordination. Intensive Care Med 20: 225-232.

10. Conference de consensus en therapeutique anti-infectieuse de la SPILF (1990) Prise en charge et prevention du paludisme d'importation a Plasmodium falciparum.. Med Mal Infect 29(Suppl. 2): S115-141.

11. Teasdale G, Jennett B (1974) Assessment of coma and impaired consciousness. A practical scale. Lancet ii: 81-84.

12. Le Gall JR, Lemeshow S, Saulnier F (1993) A new acute simplified score (SAPS II) based on a European/North American multicenter study. JAMA 270: 2957-2963.
Hopital Avicenne, Bobigny: Y. Cohen (ICU), F. Cymbalista and R. Durand (Parasitology) Hopital de Percy: B. Debien and B. Pats (ICU), T. Samson (Parasitology)

Hopital Delafontaine, Saint Denis: M. Thuong and F. Fraisse (ICU), N. Godineau (Parasitology)

Hopital Victor Dupouy, Argenteuil: G. Plantefeve and H. Mentec (ICU), F. Leturdu (Parasitology)

Hopital de l'Archet, Nice: H. Hyvernat and G. Bernardin (ICU), P. Delaunay and P. Marty (Parasitology)

Hopital Saint Antoine, Paris: JL. Baudel and G. Offenstadt (ICU); G. Belkadi and P. Roux (Parasitology)

Hopital Louis Mourier, Colombes: A. Meybeck and D. Dreyfuss (ICU), G. Galeazzi (Parasitology).

\section{Author Contributions}

Conceived and designed the experiments: FB FT MW. Performed the experiments: FB PC BM JPM EP CG FS EA YC HG AM HH JLT EF LN MW. Analyzed the data: FB FT PC BM JPM EP GG FS EA YG HG AM HH JLT EF LN CR RD JLB MW. Wrote the paper: FB FT PC BM JPM EP CG FS EA YG HG AM HH JLT EF LN CR RD JLB MW.

13. Vincent JL, Moreno R, Takala, Willats S, De Mondonça A, et al. (1996) The SOFA (Sepsis-related Organ Failure Assessment) score to describe organ dysfunction/failure. On behalf of the Working Group on Sepsis-Related Problems of the European Society of Intensive Care Medicine. Intensive Care Med 22: 707-710.

14. Heymans MW, van Buuren S, Knol DL, van Mechelen W, de Vet HC (2007) Variable selection under multiple imputation using the bootstrap in a prognostic study. BMC Med Res Methodol 7: 33.

15. Phillips A, Bassett P, Szeki S, Newman S, Pasvol G (2009) Risk factors for severe disease in adults with falciparum malaria. Clin Infect Dis 48: 871-878.

16. Newman RD, Parise ME, Barber AM, Steketee RW (2004) Malaria-related deaths among U.S. travelers, 1963-2001. Ann Intern Med 141: 547-555.

17. Dondorp AM, Lee SJ, Faiz MA, Mishra S, Price R, et al. (2008) The relationship between age and the manifestations of and mortality associated with severe malaria. Clin Infect Dis 47: 151-157.

18. Tran TH, Day NP, Nguyen HP, Nguyen TH, Tran TH, et al. (1996) A controlled trial of artemether or quinine in Vietnamese adults with severe falciparum malaria. N Engl J Med 335: 76-83.

19. Legros F, Bouchaud O, Ancelle T, Arnaud A, Cojean S, et al. (2007) Risk factors for imported fatal Plasmodium falciparum malaria, France, 1996-2003. Emerg Infect Dis 13: 883-888.

20. Hanson J, Lee SJ, Mohanty S, Faiz MA, Anstey NM, et al. (2010) A simple score to predict the outcome of severe malaria in adults. Clin Infect Dis 50: 679-685.

21. Dondorp AM, Desakorn V, Pongtavornpinyo W, Sahassananda D, Silamut K, et al. (2005) Estimation of the total parasite biomass in acute falciparum malaria from plasma PfHRP2. PLos Med 2005; 2: e204.

22. World Health Organization (2006) Guidelines for the treatment of malaria. Geneva, Switzerland.

23. Rivers E, Nguyen B, Havstad S, Ressler J, Muzzin A, et al. (2001) Early-goal directed therapy in the treatment of severe sepsis and sptic shock. N Engl J Med 345: 1368-1377.

24. Dellinger RP, Carlet JM, Masur H, Gerlach H, Calandra T, et al. (2004) Surviving Sepsis Campaign guidelines for management of severe sepsis and septic shock. Crit Care Med 32: 858-873.

25. White NJ (2003) The management of severe falciparum malaria. Am J Respir Crit Care Med 167: 673-674.

26. Morch K, Strand O, Dunlop O, Berg A, Langeland N, et al. (2008) Severe malaria and artesunate treatment, Norway. Emerg Infect Dis 14: 1816-1818. 